Meta

Journal des traducteurs

Translators' Journal

\title{
La traduction face aux nouvelles pratiques en réseaux
}

\section{Freddie Plassard}

Volume 52, numéro 4, décembre 2007

La traduction et les études de réseaux

Translation and Network Studies

URI : https://id.erudit.org/iderudit/017690ar

DOI : https://doi.org/10.7202/017690ar

Aller au sommaire du numéro

Éditeur(s)

Les Presses de l'Université de Montréal

ISSN

0026-0452 (imprimé)

1492-1421 (numérique)

Découvrir la revue

Citer cet article

Plassard, F. (2007). La traduction face aux nouvelles pratiques en réseaux. Meta, 52(4), 643-657. https://doi.org/10.7202/017690ar

\section{Résumé de l'article}

Les listes de diffusion, nouvelle fonctionnalité induite par l'usage d'Internet, permettent aujourd'hui aux traducteurs de se regrouper en réseaux de façon à partager avec leurs collègues les difficultés rencontrées dans l'exercice de la profession. Ces listes s'inscrivent donc en complément des stratégies classiques de résolution de problèmes et se substituent parfois même à certaines étapes du processus de traduction. La dimension cognitive, jusqu'à présent abordée à l'échelle individuelle, acquiert de ce fait une dimension collective, non sans incidence sur la représentation du processus de traduction.
Ce document est protégé par la loi sur le droit d'auteur. L'utilisation des services d'Érudit (y compris la reproduction) est assujettie à sa politique d'utilisation que vous pouvez consulter en ligne.

https://apropos.erudit.org/fr/usagers/politique-dutilisation/ 


\title{
La traduction face aux nouvelles pratiques en réseaux
}

\author{
FREDDIE PLASSARD \\ Université Paris 3, Paris, France \\ fplassard@libertysurf.fr
}

\begin{abstract}
RÉSUMÉ
Les listes de diffusion, nouvelle fonctionnalité induite par l'usage d'Internet, permettent aujourd'hui aux traducteurs de se regrouper en réseaux de façon à partager avec leurs collègues les difficultés rencontrées dans l'exercice de la profession. Ces listes s'inscrivent donc en complément des stratégies classiques de résolution de problèmes et se substituent parfois même à certaines étapes du processus de traduction. La dimension cognitive, jusqu'à présent abordée à l'échelle individuelle, acquiert de ce fait une dimension collective, non sans incidence sur la représentation du processus de traduction.
\end{abstract}

\begin{abstract}
As a new development of the Internet, distribution lists enable translators gathered in so-called networks to share their difficulties with their colleagues. Distribution lists therefore appear as a supplement to traditional problem-solving strategies and sometimes even as a substitute to conventional steps of the translation process. Cognitive processes considered so far on an individual basis can be tackled on a collective basis, which leads to revising established patterns of the translation process.
\end{abstract}

\section{MOTS-CLÉS/KEYWORDS}

listes de diffusion, réseau de traducteurs, cognition individuelle, socio-cognition, pratique coopérative

«Il faut sortir de l'acte individuel, qui n'est que l'embryon du langage, et aborder le fait social.»

F. de Saussure

À l'heure du foisonnement des discours relatifs aux réseaux et à l'Internet, de la «techno-utopie réticulaire» (Musso 2003), il est difficile de garder les idées claires sur les processus à la fois cognitifs, sociaux et techniques induits par l'Internet, sans verser dans l'excès, soit par adhésion immodérée à la technophilie ambiante, soit, $a$ contrario, par son rejet. Il est cependant des auteurs et des ouvrages pour nous aider à faire la part du mythe, de l'idéologie et de la réalité, et à porter sur les phénomènes sociaux et techniques et sur leurs relations un regard aussi lucide que possible. Pierre Musso, spécialiste des réseaux dans leur dimension tant technique qu'idéologique, fait, avec l'ouvrage Critiques des réseaux (2003), partie du nombre et c'est pourquoi nous avons choisi de nous en inspirer en grande part pour retracer l'historique de la notion de réseau. 


\section{Retour sur la notion de réseau}

Omniprésente dans l'éventail des disciplines, de la biologie aux sciences cognitives, en passant par la sociologie, les mathématiques, la gestion ou les sciences politiques, la notion de réseau est devenue passe-partout, au point de ne plus pouvoir prétendre, selon P. Musso ${ }^{1}$, au statut de concept, du moins dans le champ des sciences humaines, même si elle s'avère un outil d'analyse souvent utile.

\subsection{Portée du concept}

Défini tour à tour comme structure composée d'éléments en interaction liés entre eux par des chemins ou liaisons, «structure d'interconnexion instable dans le temps" et néanmoins régie par des règles de fonctionnement, le réseau est la figure même de "l'inter», avec pour substance l'intermédiaire et le passage. Figure du lien, de la transition et du passage, le réseau favorise l'interaction entre éléments et le fonctionnement de systèmes complexes. Le terme désigne à la fois l'espace tissé dans l'«inter » et le contour de cet espace intermédiaire. Le préfixe «inter» se déploie lui-même dans différentes directions: celle de l'interaction, ou des interactions verbales auxquelles donnent lieu les échanges entre internautes, ici traducteurs; celle de l'«intermédiation", sous la forme de la participation active des co-listiers sollicités pour donner leur avis sur telle ou telle question; et celle de l'«interconnexion», autrement dit la médiation technique.

Le réseau se décline sur plusieurs plans: celui, matériel, d'une ou d'infrastructures, qu'il s'agisse de chemins de fer, de réseaux de transport, de distribution d'eau, d'électricité, ou aujourd'hui de télécommunications, et celui, immatériel, mais généralement indissociable, de la gestion, de l'exploitation et du financement des réseaux matériels, et étendu à l'interaction, l'interrelation, l'intermédiation entre des agents, des fonctions et des lieux. Le réseau véhicule l'image d'une activité toujours ouverte sur la continuation, sur l'acheminement continuel de flux, y compris financiers, langagiers, de sorte que «le réseau est conçu à la fois comme une technique qui fait lien [...] et comme un opérateur politico-moral qui fait système» (Musso 2003: 206).

En tant que forme, dispositif technique et mode d'organisation, le réseau joue comme intermédiaire qui ne retient pas les flux de toute nature, transport, fluides, voix, données, mais les canalise pour en garantir le mouvement continu, fonction de régulation. Au réseau-pont, infrastructure destinée à établir le lien entre entités ou points distincts, fonction de transition, se superpose, selon Musso, le réseau-flux, quelle qu'en soit la nature, que le réseau-infrastructure a précisément pour objet d'acheminer, de faire circuler. D’un côté, le passage, la transition, de l'autre, l'immersion dans un ensemble de flux (Musso 2003: 353). Cette circulation peut aussi véhiculer, dans son versant idéologique, un rejet des structures hiérarchisées perçues comme entravant la fluidité et la circulation sociale, au profit de l'affirmation des valeurs de l'association, des relations «horizontales, fraternelles» (Musso 2003: 196) et non médiatisées dont le réseau est le symbole. Outre une application sociale, voire politique, la notion de réseau revêt aussi une dimension cognitive. 


\subsection{Réseau et cognition}

L'historique de la notion de réseau, tel que le conçoit Musso, montre qu'elle acquiert progressivement un statut épistémique qui en suscite l'application généralisée non seulement aux entités physiques, réseaux matériels, et aux entités abstraites, réseaux immatériels, mais à la représentation même des uns et des autres, autrement dit aux connaissances, c'est le cas de l'Encyclopédie de Diderot et d'Alembert, conçue et architecturée sur le modèle du réseau. Un nouvel ordre spatial mais aussi cognitif s'instaure au XVIII ${ }^{\mathrm{e}}$ siècle, où le réseau, forme générique qui "parle à la vue », devient instrument de connaissance. Formalisé, notamment par Leibniz, le réseau passe au rang d'instrument, voire de schème cognitif, avec pour avantage «de permettre une mise en forme du multiple et du complexe», d'offrir une "raison graphique» à la fois explicatrice et organisatrice du savoir et fondée sur le postulat que «voir c'est savoir» (Musso 2003: 95).

La lente montée en puissance de la notion de réseau marque son apogée avec le comte de Saint-Simon qui établit un rapport entre structure technique et forme d'organisation sociale - celle du territoire induite par les chemins de fer par exemple - autrement dit entre réseau matériel, technique, et réseau immatériel, entendu cette fois comme réseau social. Cette apogée n'est cependant que l'amorce du dévoiement de la notion, dont les saint-simoniens, disciples trop zélés de leur maître à penser, seront, selon P. Musso, les artisans. Vulgarisation outrancière, réification et fétichisation du concept se solderont par un «aplatissement» du rapport existant entre deux types de réseaux ou deux plans de réalité: infrastructure matérielle d'une part et structuration sociale de l'autre, au point de faire de la technique l'agent ou le principe même du changement social, d'établir une relation d'équivalence entre réseau technique et changement social, là où il s'agissait initialement de faire du réseau le point de passage de cette transformation (Musso 2003: 208).

À cette lecture de la pensée de Saint-Simon correspond un passage au premier plan de valeurs comme la collaboration et le consensus. Dès lors,

le réseau technique est censé réguler le social, comme le cerveau contrôle le corps humain. L'ingénieur se fait sociologue ou psychologue en naturalisant le réseau technique qu'il conçoit et fabrique. Réciproquement, les sciences sociales empruntent à l'ingénieur, pour penser les relations sociales et les organisations à l'aide de la notion de réseau. (Musso 2003: 289)

Chaque innovation réalisée sur le mode du réseau ou le mettant en jeu n'aura de cesse que de raviver cette vision réductrice, permettant d'annoncer une "révolution sociale» (Musso 2003: 233) avec la naissance de l'électricité, du téléphone, de l'ordinateur, de la télématique, du câble, du satellite ou de l'Internet, tandis que les sociologues en feront une clé de la configuration des relations sociales, dans une survalorisation de la médiation sous toutes ses formes et un déterminisme de la relation et de l'interaction. Ce mouvement trouve son apogée, selon P. Musso, dans la «techno-utopie cyberspatiale», fondée sur l'assimilation du fonctionnement du cerveau à celui d'un ordinateur, sur la mise en réseau des uns et des autres, et la déduction qu'il est possible d' «interconnecter» les cerveaux humains au même titre que les ordinateurs à l'échelle planétaire (Musso 2003: 330). Avec cette étape se parachève à ses yeux la dégradation de l'usage d'une notion d'abord réifiée puis réduite à une «technologie de l'esprit», entendue comme procédé stéréotypé de raisonnement. 
Le réseau, notion devenue passe-partout, «précepte et décept» (Musso 2003: 290), finit par n'être plus qu'un mot de passe pour désigner des relations ou interactions entre des éléments quelconques, synonyme d'intermédiaire, d'interconnexion et de passage, sans pour autant cesser d'être le support d'une idéologie et d'un véritable culte (Musso 2003: 19).

\subsection{Application aux réseaux de traducteurs}

Ce bref historique de la notion de réseau, de ses implications théoriques, n'était toutefois qu'un tremplin pour passer à l'examen des listes de diffusion de traducteurs, contrepartie virtuelle - parce que directement liée au support numérique - de réseaux professionnels parfois préexistants, comme le réseau franco-allemand de traducteurs qui servira de référence au présent article.

Si la notion même de réseau suscite des controverses théoriques, cette dénomination relève d'un choix délibéré de la part des groupements de traducteurs, choix que certains des éléments définitoires proposés par P. Musso ne peuvent que conforter. La liste de diffusion, dispositif numérique permettant les échanges à distance entre membres du réseau considéré, prend ici le relais de groupes de travail constitués notamment dans les années 1990 à l'initiative des traducteurs, de façon à faciliter les échanges, à mutualiser réflexions et travaux en prise sur la réalité professionnelle tout en créant un cadre d'affiliation. L'apport de la médiation technique s'apprécie donc en termes de fréquence et de facilité d'échanges auparavant tributaires de l'éloignement géographique et du rythme nécessairement très espacé des rencontres regroupant une bonne cinquantaine de personnes, là où le réseau virtuel en regroupe une centaine.

Le terme de réseau désigne donc le groupe constitué, "espace de l'inter» dont l'accès est soumis à conditions, en vertu d'un «recrutement» transversal, fondé sur la combinaison linguistique pratiquée en traduction, outre l'adhésion au syndicat professionnel de tel ou tel pays de langue allemande ou française. Tout en exerçant la profession sous différents statuts, y compris le statut libéral qui implique de fait une situation de concurrence, les traducteurs de ce réseau, mais aussi de nombreux autres du même type, semblent tirer meilleur parti du partage de leurs difficultés et des pratiques de coopération et d'entraide que de la défense strictement individuelle de leur territoire ou de leurs intérêts respectifs. La dimension «matérielle» du réseau tient ici aux flux d'information tangibles échangés par les personnes physiques, tandis que la dimension immatérielle tient à la finalité du réseau, celle de la mise en circulation de ces mêmes flux à des fins professionnelles. Le «réseau » réuni sur une liste de diffusion relève à la fois du réseau-pont, dans la mesure où il constitue une infrastructure, même virtuelle, à laquelle ont recours les traducteurs en complément des outils classiques de résolution des problèmes de traduction, dictionnaires, documentation en ligne et autres, du reste mobilisés ou mentionnés dans les échanges, mais aussi du réseau-flux, dans la mesure où les échanges suscités par les traductions et les traductions elles-mêmes constituent des flux. La distinction opérée par J.-C. Beacco (1995: 140) à propos des communautés translangagières, entre flux internes et flux externes, est ici aisément transposable. Entrent dans la catégorie des flux internes les productions textuelles à usage interne que sont les messages échangés entre co-listiers, et dans celle des flux externes les traductions, textes initialement 
produits par des scripteurs extérieurs au groupe et destinés in fine à un cercle de lecteurs eux aussi extérieurs au réseau réunis par la liste de diffusion.

\section{Réseau-pont: les listes de diffusion sous l'angle des interactions verbales}

Le matériau verbal fourni par les listes de diffusion de traducteurs se prête à différentes analyses, traductologique d'une part, abordée ici au titre du réseau-flux (voir infra), mais offre aussi une dynamique propre analysable en termes d'interaction verbale et de pratique coopérative, impliquant peut-être de déborder le cadre strictement traductologique. Les travaux de C. Kerbrat-Orecchioni (1990: 77 et suivantes), permettent sur ce point de dégager plusieurs paramètres utiles à la description de ces listes. Les interactions verbales se caractérisent en premier lieu par leur cadre qui englobe à la fois la configuration physique de l'espace de communication, les participants et leur statut participatif, la finalité, le type d'interaction, les moyens à disposition et les règles du jeu.

\subsection{Cadre spatio-temporel de l'interaction}

Ce cadre, virtuel en tant que subordonné au support numérique, n'en délimite pas moins un espace régi par des conditions d'adhésion déjà mentionnées, dont une inscription, mais aussi le respect des règles de la nétiquette, vade-mecum du savoirvivre des communautés virtuelles, constituée des "prescriptions et proscriptions conventionnelles connues de tous les membres compétents de la communauté» (Cosnier 1984: 215). Un suivi sur trois mois (voir Annexe), nous a permis de noter le nombre de messages échangés par question et par mois ainsi que leur date. La durée totale d'un échange ne dépasse pas, en règle générale, deux à trois jours. Des réponses n'en continuent pas moins à affluer et relancer parfois la discussion, même après clôture apparente d'une séquence, comme on le verra dans le second exemple (voir infra, $\$ 3.2$ ).

\subsection{Cadre participatif}

Une des particularités des interactions verbales sur les listes de diffusion tient au nombre de participants potentiels. Chaque message étant adressé par courrier électronique sur le mode "un vers plusieurs», tous les participants en sont a priori destinataires. Les éléments paratextuels tels que l'intitulé de la question, formulé soit par référence au domaine de connaissance, soit par mention explicite du segment textuel posant problème, soit allusivement, permettent un filtrage des messages dès réception. Il en résulte un écart entre le nombre de destinataires potentiels et celui des lecteurs effectifs, ceux qui choisissent de lire le message reçu, et enfin celui des lecteurs actifs, ceux qui réagissent au message et y répondent. Tout en continuant à relever de la structure de communication classique en pôles émission et réception, le rôle des participants y est interchangeable, à l'intérieur d'une même séquence ou d'une séquence à une autre, tandis que tout un pan du lectorat, témoin de l'échange, n'intervient pas. Sa présence implicite transparaît dans certaines réponses, comme le montrent, dans le premier exemple (voir infra, \$3.1), quelques réparties de Philippe 
qui tient à "sauver la face» envers un public indistinct mais qui le connaît bien et à qui il s'adresse indirectement sur le mode de la connivence: «[...] pour ne pas passer une fois de plus pour un vilain petit canard». D’horizons géographiques divers et pourtant soudé par une même pratique professionnelle, ce réseau social partage une «histoire conversationnelle» (Kerbrat-Orrecchioni 1990: 80) tissée de toutes les séquences préalablement échangées dans le même espace, et à laquelle il est parfois fait explicitement allusion.

La liste, fermée, est "modérée», régulée par un modérateur, type particulier de participant qui veille au bon déroulement des échanges, moyennant la centralisation et éventuellement le filtrage des messages, voire, lorsque les échanges ou débats prennent un tour trop polémique, des interventions tout à fait explicites destinées à éviter les dérives de toute nature. Ses interventions sont néanmoins rares et il semble que l'auto-discipline soit généralement respectée à l'échelle du groupe, les abonnés veillant de leur propre chef à rappeler à l'ordre les colistiers en proie à une dérive quelconque au regard de la nétiquette spécifique de la liste.

\subsection{Objet et finalité}

L'obligation de confidentialité et le support des échanges restreignent la teneur des questions posées sur le plan microtextuel, ponctuel: développement d'un sigle ou d'une abréviation, lexème ou terme, usage de mots ou formules, faux amis et doublets, traduction attestée d'une citation, énumération non exhaustive sur laquelle peut se greffer, à l'occasion, un débat sur des questions non plus ponctuelles mais de fond comme l'anglicisation des langues, la féminisation des titres et bien d'autres. Compte tenu du profil des traducteurs, spécialisés le plus souvent, et travaillant pour le compte d'entreprises ou d'institutions en prise sur des réalités tout à fait contemporaines, la liste est un bon observatoire de la langue non seulement usitée, mais aussi créée, au jour le jour, et la néologie n'y est pas rare. Il s'agit d'un écrit hâtif, fonctionnel, ce qui n’empêche ni la technicité des réponses, leur précision, les références citées à l'appui d'un propos, ni les controverses théoriques prolongées auxquelles donnent parfois lieu certaines questions.

La liste, à vocation épistémique, fonctionne sur un mode mutualiste puisqu'il s'agit de mettre en commun non seulement les difficultés rencontrées dans la pratique mais aussi les moyens et stratégies permettant de les résoudre. Elle est l'outil d'un réseau socio-professionnel, d'une communauté de pratique à la fois virtuelle, support informatique oblige, et réelle, comme précédemment évoqué (voir supra, $\$ 1.3$ ). Cadre physique, la liste importe par sa finalité, la coopération professionnelle, finalité première par rapport à laquelle le caractère virtuel peut sembler secondaire ou instrumental, même s'il induit par sa nature même une forme et, surtout, une fréquence spécifiques d'échange.

\subsection{Nature de l'interaction}

Selon la catégorisation proposée par C. Kerbrat-Orecchioni, le «texte conversationnel» ou flux interne qui se tisse sur les listes de diffusion relève d'une interaction verbale qui peut être dite «focalisée», puisque les participants s'adressent la parole et que le groupe se structure autour d'un «foyer» commun sur lequel se concentre 
l'attention intellectuelle et visuelle des différents participants (Goffman 1974: 117). Cette précision s'applique ici à une réserve près: l'attention effectivement focalisée ne l'est que par texte interposé, à lire sur écran, et non en co-présence physique des participants. Cet état de fait conforte pour ainsi dire au second degré le constat formulé par H. Buzelin (2004: 731), selon lequel les traductologues interrogent avant tout des représentations écrites.

À l'intérieur de la catégorie «interaction verbale», les flux internes des listes de diffusion relèvent de prime abord de la discussion, dans la mesure où la composante argumentative y prévaut et où les partenaires en présence s'efforcent de se convaincre les uns les autres (Kerbrat-Orecchioni 1990: 118). On le verra dans le premier exemple, où les différents points de vue exprimés finissent par converger sur une même solution, dûment argumentée, explicitée et de ce fait convaincante. Elle n'en présente pas moins certains traits de la conversation, au sens non pas d'un "archilexème recouvrant toute la gamme des échanges verbaux", mais d'un type particulier d'interaction verbale, de caractère immédiat dans le temps et l'espace, familier et non formel où tous les participants sont a priori présents au même titre, quelles que soient les tentatives, ici ou là, de s'arroger une place dominante et les mécanismes plus subtils d'assignation de places dans cet espace social. Le seul trait qui fasse exception à la définition de la conversation est celui de la gratuité, l'échange étant en règle générale finalisé, doté d'une visée instrumentale, celle de répondre à une question précise, hormis les cas dits ici de «socialité» (voir ci-dessous, $\$ 3$ ).

\section{Réseau-flux : exemples d'échanges sur les listes de diffusion}

Les flux externes - traductions - restant pour ainsi dire «hors champ», en raison de leur confidentialité, mais aussi des contraintes notamment temporelles qui ne permettent de soumettre à délibération que des extraits textuels de l'ordre du segment, seuls les flux internes peuvent être réellement observés sur les listes de diffusion.

La distinction entre processus de traduction et produit, traduction-texte, quoique à nos yeux partiellement arbitraire - le processus n'a de raison d'être que centré sur l'élaboration du produit et le «produit» complexe qu'est la traduction ne peut s'élaborer qu'au terme d'un processus -, n'en permet pas moins de catégoriser en première approche les questions posées en fonction du stade du processus dont elles relèvent, même s'il y entre une part de conjecture en l'absence, le plus souvent, de précisions de la part des traducteurs sur ce point. L'emprunt de notions traductologiques à la théorie interprétative de la traduction nous permettra de décrire ces échanges verbaux sous un angle familier. C'est à partir de la nature de la question posée, d'ordre ponctuel, et d'une catégorisation même sommaire, que le stade en question peut être déduit. On peut ici distinguer celui de la Compréhension pour les questions portant sur l'analyse et l'appropriation du texte de départ, de la Reformulation pour celles qui relèvent de la réexpression, en langue d'arrivée, du produit de la compréhension, de la Terminologie, lorsqu'il s'agit de cerner une notion et son équivalent en langue d'arrivée, de la Recherche documentaire lorsqu'il s'agit d'aiguiller l'exploration d'un domaine de connaissance ou d'affiner des connaissances préalables, et enfin de la «Socialité» lorsque la question posée, sans rapport direct avec la traduction, donne lieu à un échange qui relève de la fonction phatique du langage et témoigne de la fonction de médiation sociale de la liste, relais d'une communication qui n'aurait pas 
lieu sans ce support. On pourrait sans doute objecter que toutes les questions relèvent de cette catégorie, puisque la dimension sociale est présente d'entrée de jeu, inhérente au principe même des listes de diffusion. Disons que les questions relevant de cette catégorie font primer le lien social sur toute autre dimension, notamment cognitive, que le lien social y est cultivé en tant que tel. Cette ébauche de catégorisation est souvent mise à mal par les exemples traités: une même question, censée porter sur une unité terminologique, peut en réalité relever et de la terminologie, du moins dans la solution qui lui sera fournie, et de la compréhension, si la difficulté tient en réalité à une connaissance trop approximative de la notion en langue de départ, et même de la reformulation, s'il faut intégrer la notion au contexte verbal dans la langue d'arrivée. Voyons plutôt quelques exemples choisis pour leur représentativité, tant par la nature de la question posée que par la dynamique de l'échange suscité.

\subsection{Premier exemple: Lehre}

\section{Question :}

Bonjour à toutes et à tous,

Y a-t-il parmi vous des spécialistes de la traduction de brevets allemands en français? Dans la description d'un brevet allemand, j'ai du mal à traduire le passage suivant: «Im Anspruch 6 ist angegeben, dass.... Diese Lehre ist konträr zu der der DE-...(réf. du document) entnehmbaren Lehre».

Comment traduire la $2^{\mathrm{e}}$ phrase et en particulier «entnehmbare Lehre»?

Merci d'avance pour vos suggestions. Cordialement, Sylvie

\section{$1^{\text {re }}$ réponse}

Bonjour Sylvie, [Reprise du libellé de la question] Pourquoi des brevets? ?? :-)))

Eine «entnehmbare Lehre»... c'est un gabarit amovible... Bien confraternellement, Philippe

\section{$2^{\mathrm{e}}$ réponse}

Étonnant, hier soir j'avais le message de réponse de Philippe, mais pas la question de Sylvie et je m’adresse à la bonne personne: vous avez écrit [Reprise de la réponse 1]. Je pencherais pour une autre explication: «Il est indiqué dans la revendication 6 que ce gabarit est contraire au gabarit stipulé (ou mentionné ou figurant) dans la/le DE-... (réf. du document).»

À propos de brevets, la communication sur les brevets dans la dernière édition de Traduire est très intéressante, même si elle se penche surtout sur les brevets anglais. Les règles des brevets sont impénétrables - et relativement inamovibles!!! et ce dans toutes les langues, semblerait-il!!

Cordialement, Claudie

\section{$3^{\mathrm{e}}$ réponse}

Bonjour Sylvie, Bonjour Philippe,

Der von Philippe vorgeschlagene franz. Ausdruck bezieht sich auf eine mechanische Lehre im Sinne einer abnehmbaren Schablone.

Dazu nur kurz eine Bemerkung: Da hier kein ausführlicher Kontext hinsichtlich des Patents angegeben ist, ist evtl. auch noch eine andere Bedeutung denkbar. Die Lehre im Sinne der Lehre zum technischen Handeln etc.). Die aus dem Patentanspruch hervorgehende Lehre (eng. teaching) kann auch im Widerspruch zu einer Lehre eines anderen Patents stehen. Dieser Anspruch wahrscheinlich dann zu prüfen. Welche 
Bedeutung hier gemeint ist, geht sicher aus dem Patentgegenstand hervor. Dies nur als Kommentar. Die entsprechende franz. Übersetzung finden dann sicher die Muttersprachler. Bien confraternellement, Winfried

\section{$4^{\mathrm{e}}$ réponse}

Bonjour Claudie, [Reprise de la $2^{\mathrm{e}}$ réponse]

Oui, mais Winfried a raison aussi et c'est sûrement lui qui détient la clé. Je m'étais arrêté à un aspect purement technique. Mais à bien relire la phrase (tronquée), il y a effectivement une forte chance qu'il s'agisse d'un "enseignement à tirer»... On ne soulignera jamais assez la grande importance du contexte. Mais dans le cas présent, il y a une autre réflexion qui me taraude... mais dont je m'abstiendrai... pour ne pas passer une fois de plus pour un vilain petit canard.

Bien confraternellement, Philippe

\section{$5^{\mathrm{e}}$ réponse}

Bonjour à tous,

Je ne suis pas spécialiste des brevets et ne sais bien sûr pas sur quel sujet porte celui que vous traduisez, mais une "Lehre", avant d'avoir la signification technique de gabarit, a celui de théorie, doctrine, position de principe, laquelle se lit - ou ressort vraisemblablement du document mentionné. Freddie

\section{$6^{\mathrm{e}}$ réponse}

Bonjour Freddie, [Reprise de la réponse 5]

Tout à fait d'accord... Depuis le message que tu cites, nous sommes allés beaucoup plus loin dans la réflexion...:-)) Bien confraternellement, Philippe

\section{Récapitulatif}

Merci à toutes celles et ceux qui m’ont adressé leurs suggestions et explications.

«Diese Lehre ist konträr zu der der DE(...) entnehmbaren Lehre» = Cet enseignement est contraire à l'enseignement fourni par le document DE (...). Cordialement, Sylvie

Dans cette séquence, les réponses ne s'adressent pas toutes à la traductrice qui a posé la question initiale, et quoique envoyées à tous les colistiers, constituent souvent une réponse individuelle au locuteur précédent, presque en aparté, entraînant une trame complexe des échanges, selon la structure suivante:

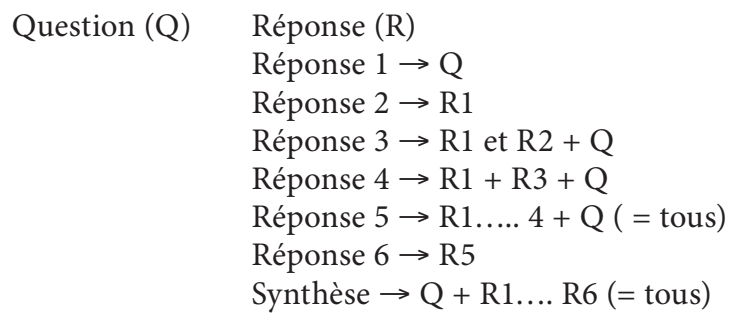

\subsubsection{Analyse de la séquence}

Réduite en apparence à deux mots, la question est présentée comme une difficulté de reformulation, mais relève aussi, comme le montrera l'échange suscité, de la compréhension. Elle met d'une part en question la «bonne lecture» de l'expression «entnehmbare Lehre» dans son contexte verbal, la phrase tronquée où elle apparaît, et "générique», le genre textuel, brevet, susceptible d'induire certaines stéréotypies 
énonciatives, et sollicite d'autre part une reformulation: de la juste compréhension découlera une reformulation contextuellement pertinente. On peut noter dès ce stade le choix des éléments fournis aux colistiers, jugés suffisants pour susciter des réponses. Seuls quelques éléments suffisent à orienter la compréhension, ce qui dénote la part de la «suppléance mentale» à l'œuvre dans l'élaboration de toute traduction, permettant, à partir de quelques indices de contextualisation, d'intégrer l'élément problématique à un schéma cognitif résultant d'une synthèse entre les éléments fournis dans le libellé de la question et les connaissances antérieures des lecteurs. La question est en réalité d'ordre référentiel: à quoi fait référence le mot Lehre?

La première réponse est formulée par un lecteur tellement expert qu'il a lu trop vite et, tout en tenant compte des indices de contextualisation fournis, a procédé par inférence de liaison, en mobilisant hâtivement un schéma cognitif auquel il a associé des correspondances lexicales, Lehre/gabarit, entnehmbar/amovible, en une sorte d'activation réflexe de son «dictionnaire mental». La deuxième réponse embraye sur l'échange déjà engagé, tout en proposant une reformulation qualifiée d' "explication» qui conforte la traduction de Lehre par gabarit, mais fait état d'un doute sur l'acception concrète de entnehmbar, «déstabilise» le locuteur précédent tout en élargissant le propos. La troisième, celle d'un traducteur de langue maternelle allemande, est à la fois une "rétro-traduction» de la première réponse, où il remplace Lehre par un synonyme, Schablone, et une paraphrase intra-linguistique de l'énoncé posant question. Il explicite ce faisant l'énoncé de départ, quitte à recourir à un équivalent anglais, lingua franca censée aplanir les difficultés de compréhension, et attire l'attention sur les limites du «contexte» fourni, l'objet même du brevet (Patentgegenstand), autrement dit le domaine, n'ayant pas été précisé. Le lexème Lehre est à interpréter ici dans un sens non pas technique mais beaucoup plus général d'«enseignement à tirer», de "leçon». Par cette réponse, le traducteur réoriente la compréhension de l'énoncé de départ, et notamment celle de Lehre. La quatrième réponse, formulée par le même locuteur que la première, renforce la troisième réponse. Il n'hésite pas à recourir à certaines astuces pour faire oublier son erreur initiale et susciter la curiosité, dans une relance de la lecture. La cinquième réponse fait entrer en scène un nouvel interlocuteur qui renforce à son tour les réponses précédentes, quoiqu'un peu tard. La réponse, quoique pertinente, perd de son intérêt du simple fait d'avoir déjà été formulée. La sixième réponse, également formulée par le même locuteur que la première réponse, fait valoir la progression de la discussion. C'est la dimension «socio» plus que cognitive qui est ici mise en avant; l'auteur qui s'était affiché un peu rapidement d'entrée de jeu peut ainsi retrouver la face aux dépens d'un tiers retardataire. Le récapitulatif fourni sous la forme de remerciements indique la solution retenue, laquelle dénote une intégration cognitive des réponses reçues en une reformulation. Il y a à la fois coopération et collaboration ${ }^{2}$, dans la mesure où la première solution, trop évidente, est écartée, et où sont mises à profit les connaissances ou compétences respectives des différents locuteurs de même que leur lecture respective du même énoncé. C'est de la confrontation de ces différentes lectures que pourra émerger la compréhension juste de l'énoncé de départ, elle-même source d'une reformulation adéquate en langue d'arrivée.

Au fil de cet échange, il est loisible d'observer l'infléchissement de la compréhension qu'induit le contexte. Pris au sens large de situation, de circonstances d'énonciation, ici ramenées au genre textuel des brevets, le contexte infléchit la compréhension 
sur la piste du «contenu sémantique» de l'expression à traduire, sur son référent technique, gabarit. Pris a contrario au sens strict de contexte verbal, il oriente paradoxalement la compréhension sur la piste générique, l'indication du genre textuel brevet comme inductrice de certaines stéréotypies énonciatives à identifier au stade de la lecture, et notamment la référence à un corpus théorique, Lehre, «doctrine», vraisemblablement issue d'autres brevets déposés sur le sujet et faisant avec le brevet considéré «intertexte», «interdiscours » auquel se reporter. Autrement dit, les mêmes indices de contextualisation peuvent orienter la compréhension sur des voies différentes et, même a priori suffisants, n'empêchent pas nécessairement les lectures divergentes, voire erratiques: il peut y avoir deux ou plusieurs compréhensions d'un même énoncé particularisé par un contexte, dont seule une résiste toutefois à l'analyse et à une lecture attentive. Ce qui est en question, ce n'est donc pas tant ce que le contexte cognitif et verbal fournit ou ne fournit pas, que l'amorçage qu'il induit et les connaissances qu'il active chez le lecteur, et l'aiguillage de la compréhension qui en résulte. Si un indice textuel ou contextuel induit une activation cognitive, rien ne permet de préjuger a priori de l'adéquation des connaissances activées, que le contexte verbal vient conforter ou démentir. Passons à un deuxième exemple.

\subsection{Deuxième exemple: citation d'Einstein}

\section{Question}

Bonjour à tous,

J'ai eu beau chercher partout sur Internet, je ne trouve pas l'équivalent de la citation suivante:

"Alles was denkbar ist, ist machbar». Quelqu'un connaît-il cette citation? Le plus approchant que je trouve est «L'imagination est plus importante que le savoir», mais à mon avis, ça ne colle pas vraiment... Merci de toute suggestion, Françoise 1

\section{$1^{\text {re }}$ réponse}

Bonjour Françoise,

Ma suggestion, si tu ne retrouves pas la traduction de la citation: «Tout ce qui est concevable est faisable». Cordialement, Annick

\section{$2^{\mathrm{e}}$ réponse}

Bonjour Françoise,

Même si le concept est en soi différent, est-ce que dans ton contexte: «vouloir c'est pouvoir» pourrait convenir? Si l'on extrapole, cela ressemblerait au «impossible n'est pas français", attribué à Napoléon!

Bon courage - Très cordialement, Françoise 2

\section{$3^{\mathrm{e}}$ réponse}

Bonjour Françoise,

Je trouve dans le Petit Robert, à Einstein, le titre d'un essai qu'il a écrit en 1934, "Comment je vois le monde»: à tout hasard, ta citation pourrait figurer dans cet essai. Je ne sais comment y accéder, mais c'est peut-être une piste... Claudie

\section{Récapitulatif}

Merci Claudie pour ta suggestion! Après demande de précision au client, il accepte que je donne «ma» version en ajoutant «d'après Einstein» et non plus seulement «Einstein». 
J'ai donc opté pour la formule «Tout ce qui est concevable est réalisable». Je la trouve un peu trop «traduite» et je préférerais la version donnée par Françoise 2 «Vouloir c'est pouvoir» certes un peu différente de sens, mais avec un impact tellement plus fort (c'est pour une présentation ppt pour une formation sur l'environnement). Mais étant donné que la photo d'Einstein figure juste au-dessus de la citation, je ne peux pas m'en éloigner trop.

Merci à tous ceux et toutes celles qui ont répondu à mon appel! Françoise 1

\section{$5^{\mathrm{e}}$ réponse}

À propos d'Einstein, pour ceux/celles qui sont intéressés

Guck mal, Einstein am 14.3... <http://www.einstein.ulm.de/veranstaltungen/set. html>.

Annegret

\subsubsection{Analyse de la séquence}

Toutes les réponses fournies étant adressées à la traductrice qui a posé la question, l'échange suit une trame simple où toutes les réponses convergent vers la question et qu'il n'est dès lors pas nécessaire de présenter sous forme schématique. La question porte ici sur l'existence ou non d'une traduction attestée de propos attribués à Einstein, et met en cause le «déjà traduit» sous la forme de l'accès à cette éventuelle traduction mais aussi de l'obligation ou non de reprendre un éventuel libellé préexistant. Localiser cette citation isolée de son contexte d'origine, non précisé, relève de l'exploit, sauf à avoir accès au corpus numérisé de l'intégralité de l'œuvre d'Einstein, pour autant qu'il existe. Reste à savoir dès lors comment traduire la citation, en l'absence d'accès au texte où elle est puisée et a fortiori à sa traduction. Les deux premières réponses sont des propositions de traduction par équivalence, en fonction bien sûr du sens de la citation mais aussi de l'adéquation au contexte en langue d'arrivée, et non par référence à une traduction publiée. La troisième réponse ne donne pas une "solution», mais une piste de recherche éventuelle qui implique une transposition des stratégies de recherche documentaire ordinairement mobilisées à un type de corpus relativement peu exploré dans ce champ de la pratique professionnelle, les textes d'auteur. La suggestion est bien accueillie mais la solution adoptée en définitive est un compromis trouvé avec le donneur d'ouvrage, sous la forme d'une adaptation «d'après Einstein », solution jugée néanmoins « un peu trop traduite», autrement dit encore trop proche de la formulation de départ. La mention d'un site de nature à élargir l'horizon de recherche est apportée à titre de complément d'information, après formulation du récapitulatif, et incite à une relance de l'échange et de la recherche.

Les deux séquences observées sur la liste de diffusion du réseau franco-allemand nous ont donné accès à l'atelier non pas d'un mais de plusieurs traducteurs et à la mise en délibération collective de certains points de traduction. La multiplication des listes de diffusion et la fréquence des échanges qui s'y livrent portent à penser qu'il s'agit là d'une tendance vraisemblablement durable induite par l'usage des technologies de l'information et par l'accélération des cadences de travail qui vont de pair, ne laissant que fort peu de temps à consacrer à des démarches en soi généralement longues, comme la recherche documentaire par exemple. Ce que les listes de diffusion apportent aux réseaux ou "groupements d'intérêt cognitif» pré-existants ne tient donc pas tant au principe de l'échange de connaissances, déjà mis en œuvre auparavant, qu'à la démultiplication de leur volume et de leur fréquence, au point 
que la consultation des pairs est désormais parfaitement intégrée à la pratique professionnelle quotidienne, à en juger non pas par des statistiques à ce jour non établies, du moins par nos soins, mais par différents facteurs, dont les inquiétudes formulées par certains co-listiers intempestivement déconnectés, par la fréquence d'occurrence des mêmes noms sur une même liste en l'espace de quelques jours ou semaines et par la nature et le nombre de questions posées et de réponses apportées et dont la liste considérée ici n'est pas nécessairement la plus représentative.

Les «flux internes» constitutifs de ces échanges peuvent parfois donner l'impression d'un bricolage intellectuel, dans la mesure où il s'agit de parer au plus pressé, de trouver un compromis, soit entre les différentes réponses fournies, soit avec le donneur d'ouvrage, tâtonnement qui semble aller à l'encontre des représentations bien structurées du processus de traduction, véhiculant à des fins notamment pédagogiques l'image sans doute idéalisée d'un processus raisonné et maîtrisé de bout en bout par ceux qui le réalisent. Ils présentent néanmoins pour avantage, par rapport à bien des méthodologies adoptées à ce jour en traductologie, de ne pas porter un regard rétrospectif sur le processus de traduction, de ne pas dissocier la traductionprocessus de la traduction-texte, certes réduit - et c'est là que le bât blesse - à un segment, et de mettre en lumière les questions que se posent réellement les traducteurs, sans aucune intervention externe, sous la forme de protocole de verbalisation par exemple. En ce sens, ces flux éclairent des «aspects également constitutifs du processus traductionnel (donc essentiels à sa compréhension), mais qui ne s'affichent pas comme tels dans le produit fini et s'effacent avec le temps: les négociations entre les agents, les imprévus et les stratégies de persuasion» (Buzelin 2004: 738). Ils montrent aussi l'intérêt qu'il peut y avoir pour les praticiens à confronter leur point de vue, à remettre en question leur propre compréhension, à obtenir l'aval de leurs collègues en cours de traduction.

Nul doute que ces nouvelles pratiques ne suscitent l'intérêt du traductologue et ne viennent enrichir la palette méthodologique à sa disposition et illustrer les négociations, tensions et complicités dont se tisse en amont le "produit fini» qu'est la traduction. Ainsi s'ouvre à ses yeux un champ d'investigation permettant d'éprouver les modèles existants, voire de procéder à un ajustement de la description du processus de traduction. Savoir apprécier l'incidence réelle des pratiques en réseau sur le déroulement de ce processus et sur la traduction des segments soumis à délibération d'une part et intégrer d'autre part à cette description la dimension sociale des processus cognitifs à l'œuvre, telles sont sans doute les voies de recherche à approfondir, quitte à interroger une fois de plus et sous un jour nouveau l'articulation du palier individuel de la compréhension et de la production de la parole au palier collectif de l'usage linguistique, autant de bornes posées à la pratique de la traduction.

\section{NOTES}

1. Initialement chercheur au Centre national d'études des télécommunications (CNET) puis membre du conseil d'administration de France Télécom, Pierre Musso, philosophe, est aujourd'hui professeur de sciences de l'information et de la communication à l'Université de Rennes et auteur de plusieurs ouvrages relatifs aux réseaux. L'idéologie actuelle des réseaux est issue selon lui d'une dichotomie entre acteurs et décideurs techniques d'une part et idéologues de l'autre, et d'un héritage dévoyé de la pensée de Saint-Simon dont il est également spécialiste.

2. Jacques Perriault (2002: 137) distingue la coopération, relation qui permet à chacun des participants d'un groupe de faire fructifier ce qu'il apporte, de la collaboration, relation de réciprocité impliquant 
une confiance suffisante envers autrui pour accepter d'apprendre de lui «dans un environnement non compétitif ", précision dont on notera le côté paradoxal. L'« environnement compétitif», établi par la situation de concurrence effective où se trouvent les traducteurs, se manifeste sur les listes de diffusion non pas par une rétention d'information, mais au contraire par son «affichage» sous la forme d'une mise à la portée de tous d'éléments d'information ou de connaissances, le plus «compétitif» étant non seulement celui qui donne la réponse juste mais sait aussi l’argumenter.

\section{RÉFÉRENCES}

BEACCO, J.-C. (1995): «À propos de la structuration des communautés discursives: beaux-arts et appréciatif», in BeACco, J.-C. et S. Moirand (dir.), Les enjeux des discours spécialisés, Les carnets du Cediscor 3, Paris, Presses de la Sorbonne Nouvelle, pp. 135-157.

Buzelin H. (2004): «La traductologie, l'ethnographie et la production des connaissances », Meta, 49-4, pp. 729-746.

Cosnier, J. (1984): «La psychanalyse, le langage et la communication», Psychothérapies 4, pp. 215-221.

Goffman, E. (1974): Les rites d'interaction, Paris, Minuit.

Kerbrat-Orecchioni, C. (1990): Les interactions verbales, Paris, Armand Colin, tome 1.

Lederer, M. (1994): La traduction aujourd'hui. Le modèle interprétatif, Paris, HachetteRéférences.

Musso, P. (2003): Critique des réseaux, Paris, PUF, coll. «La politique éclatée».

Perriault, J. (2002): L'accès au savoir en ligne, Paris, O. Jacob, coll. «Le champ médiologique».

\section{ANNEXE}

\section{Suivi des échanges sur la liste du réseau franco-allemand pendant trois mois}

Catégorisation:

Compréhension: problème de compréhension en langue de départ

Reformulation: problème de reformulation en langue d'arrivée

Terminologie: cerner un concept et lui trouver une correspondance (en langue)

Recherche documentaire: élargir le champ de recherche notionnel ou intertextuel

Socialité: question sans rapport direct avec la traduction, fonction phatique des échanges

\begin{tabular}{|l|l|c|c|}
\hline Sujet (intitulé) & Catégorie & Nbre Mges & $\begin{array}{c}\text { Date } \\
(2005)\end{array}$ \\
\hline Druck & Terminologie & 5 & $08 / 01$ \\
\hline Potentiel flottant & Compréhension + Reformulation & 3 & $08 / 01$ \\
\hline Für's dicke Fell & Reformulation (publicité) & 9 & $11 / 01$ \\
\hline Zitat und Imitat & Reformulation & 7 & $11 / 01$ \\
\hline Strommix & Terminologie & 7 & $24 / 01$ \\
\hline Normen & $\begin{array}{l}\text { Compréhension (Recherche } \\
\text { documentaire) }\end{array}$ & 8 & $26 / 01$ \\
\hline Décroissance & Terminologie & 4 & $28 / 01$ \\
\hline Lehre & Compréhension & 8 & $29 / 01$ \\
\hline $\begin{array}{l}\text { Demande d'immersion en } \\
\text { Allemagne }\end{array}$ & Socialité & 17 & $29 / 01$ \\
\hline \hline Hohe Feiertage & $\begin{array}{l}\text { Compréhension (Bagage } \\
\text { cognitif d'ordre culturel) }\end{array}$ & 5 & $02 / 02$ \\
\hline Fürsorgeverpflichtung & Reformulation & 6 & $03 / 02$ \\
\hline $\begin{array}{l}\text { Ruhiges Produktmässiges } \\
\text { Erscheinungsbild }\end{array}$ & Reformulation & & \\
\hline
\end{tabular}




\begin{tabular}{|l|l|c|c|}
\hline Contexte électricité & Terminologie & 11 & $15 / 03$ \\
\hline A titre irréductible & Reformulation (Phraséologie) & 2 & $15 / 02$ \\
\hline Anhydre Medikamente & Terminologie & 4 & $16 / 02$ \\
\hline Energies renouvelables & Reformulation & 11 & $17 / 02$ \\
\hline Umlauftitel & Compréhension + Reformulation & 2 & $18 / 02$ \\
\hline SOS allemand général & Reformulation (usage) & 10 & $19 / 02$ \\
\hline Solarthermie & Compréhension + Reformulation & 5 & $22 / 02$ \\
\hline GZ & Compréhension (Abréviation) & 5 & $23 / 02$ \\
\hline Bekennender Kunde & Reformulation & 9 & $25 / 02$ \\
\hline Terme de chimie & Terminologie & 4 & $28 / 02$ \\
\hline \hline $\begin{array}{l}\text { Niedrigschwelliges.../ } \\
\text { flächendeckend }\end{array}$ & Reformulation & 8 & $01 / 03$ \\
\hline Reformstau & Reformulation & 8 & $02 / 03$ \\
\hline Erdgas (Rheinschiene) & Reformulation & 6 & $06 / 03$ \\
\hline Vie du réseau & Socialité & 8 & $07 / 03$ \\
\hline Knochenarbeit & Reformulation (publicité) & $08 / 03$ \\
\hline Beau comme un camion & Reformulation (Stéréotypie) & 4 & $09 / 03$ \\
\hline In den Gassen landen & Compréhension + Reformulation & 5 & $09 / 03$ \\
\hline Warenkreditversicherung & Reformulation & 10 & $10 / 03$ \\
\hline Repli communautaire & Reformulation & 5 & $10 / 03$ \\
\hline Citation d'Einstein & Reformulation & 6 & $11 / 03$ \\
\hline SOS allemand luxembourgeois & Compréhension (explicitation) & 6 & $11 / 03$ \\
\hline $\begin{array}{l}\text { Information (série d'articles } \\
\text { parus dans Le Monde) }\end{array}$ & Socialité (information) & 5 & $16 / 03$ \\
\hline Trojan-phish & Socialité (annonce d'un virus) & 5 & $16 / 03$ \\
\hline Gleisrot & Reformulation (équivalence) & 5 & $18 / 03$ \\
\hline Rapport d'expertise suisse & Compréhension & 5 & $20 / 03$ \\
\hline
\end{tabular}

